\title{
Do anesthesia emergence times reflect resident training progression, educational competency, and operating room efficiency?
}

\section{Luke Fitzgerald Miles}

Drexel University College of Medicine

Christopher Ryan Hoffman ( $\triangle$ CRHoffman@gmail.com )

Hahnemann University Hospital https://orcid.org/0000-0001-8387-1060

Janeway Granche

Drexel University

\section{Michael Stuart Green}

Drexel University College of Medicine

Research article

Keywords: Health Resources; Resource Allocation; Graduate Medical Education; Educational Measurement; Anesthesia, ACGME Core Competencies, Systems-based practice

Posted Date: August 30th, 2019

DOI: https://doi.org/10.21203/rs.2.13730/v1

License: (9) This work is licensed under a Creative Commons Attribution 4.0 International License. Read Full License 
Title: Do anesthesia emergence times reflect resident training progression, educational competency, and operating room efficiency?

Short Title ( $<50$ char): Does resident training impact anesthetic emergence?

\author{
Authors: \\ Luke Fitzgerald Miles MD ${ }^{1}$ \\ Janeway Granche $\mathrm{MS}^{2}$ \\ Christopher Ryan Hoffman DO ${ }^{1}$ (ORCID 0000-0001-8387-1060) \\ Michael Stuart Green DO ${ }^{1}$ (ORCID 0000-0002-0364-726X)
}

Corresponding Author

Christopher Ryan Hoffman, D.O.

Drexel University College of Medicine

Hahnemann University Hospital

245 N. 15th Street, Suite 7502, MS 310

Philadelphia, PA 19102

Telephone: 215-762-8936

Fax: 215-762-8656

CRHoffman@gmail.com

Word Counts - Abstract: 216; Manuscript: 1721

Affiliation:

1. Drexel University College of Medicine, Hahnemann University Hospital, Department of Anesthesiology \& Perioperative Medicine, Philadelphia, PA.

2. Drexel University, Department of Epidemiology \& Biostatistics, Philadelphia, PA.

Conflicts of Interest and Source of Funding:

- Funding: This study was not funded by any party.

- Conflict of Interest: All authors (Miles, Granche, Hoffman, and Green) declare that they have no conflict of interest.

- Ethical approval: This article does not contain any studies with human participants or animals performed by any of the authors. This was a retrospective review and data was de-identified.

- Informed consent: Data reviewed did not require informed consent. This was a retrospective review of operating room data and information was de-identified. 


\section{Title}

Do anesthesia emergence times reflect resident training progression, educational competency, and operating room efficiency?

\section{Abstract}

Background: Anesthesia residents are often subjectively and objectively deemed competent by their impact of cost-efficacy and perioperative metrics. Assessing knowledge acquisition and procedural skill is difficult to measure equitably. Inspecting tangible metrics of perioperative efficiency may or may not provide a source for reliable evaluation.

Methods: Retrospective case-log database review yielded 3072 surgical cases involving residents over five years. Primary variable investigated was the time from surgery completion to exit from operating room. Other variables recorded included day of week, attending anesthesiologist name, attending surgeon name, patient age, sex, American Society of Anesthesiologists physical status classification (ASA PS), and in-patient versus day surgery status.

Results: After controlling for procedure duration time, in-patient status, ASA class, surgeon, and attending anesthesiologist, resident training time became highly significant $(\mathrm{p}<0.001)$. In the fully adjusted model, one year of resident training was associated with a reduction in emergence time by 28 seconds $(95 \% \mathrm{CL}=(12,44))$. A one-hour increase in procedure time was associated with an increase in emergence time of 34 seconds $(95 \% \mathrm{CL}=(17,50))$.

Conclusions: Although a statistically significant correlation was demonstrated, the clinical significance is likely low given the relatively small amount of actual time saved. We question the 
value of utilizing perceived or measured perioperative metrics for evaluating anesthesia resident competency.

\section{Key Words:}

Health Resources; Resource Allocation; Graduate Medical Education; Educational

Measurement; Anesthesia, ACGME Core Competencies, Systems-based practice

\section{Background}

Anesthesia residency engages a multitude of educational pedagogies and training environments. This method of education is frequently difficult to accurately assess, measure, and correlate to endpoints and outcome improvement. The Accreditation Council for Graduate Medical Education (ACGME) has established six core competencies, applicable to all residency training programs. One of these core competencies, system-based practice, describes competent residents as being able to use "system resources to facilitate cost effective and safe non-subspecialty anesthesia care." [1] Delivery of cost effective, efficient care is a priority of the ACGME, hospital administrators, and policymakers alike. Efforts to improve cost efficiency may impact anesthesia resident training if education will be sacrificed at the cost of efficiency. This can potentially deteriorate the educational learning environment and impede progression to competency. In an attempt to improve efficiency, anesthesia-controlled time-related metrics have become increasingly used to assess and compare anesthesia providers. Anesthesia emergence time is a metric that is readily captured and utilized for this purpose. Prior studies have assessed the correlation between prolonged emergence (defined as $>15$ minutes) and level of anesthesia resident and demonstrated a decreased incidence of prolonged emergence as residents progressed 
through CA-1 year. [2] Some impact of new anesthesia resident trainees on anesthesia-controlled time measures are statistically significant, but may not be to an extent that results in meaningfully increased costs or decreased efficiency. [3, 4] Expanding upon this prior work, we aim to assess the relationship between level of anesthesia resident training and anesthesia emergence time, establishing an inverse correlation between level of resident training and anesthesia emergence time.

\section{Methods}

\section{$\underline{\text { Data Collection }}$}

This retrospective, observational study polled the operating room case-log database maintained by a tertiary care facility. The IRB ruled this study exempt from review. The non-emergent scheduled adult cases of every non-holiday weekday at every anesthetizing location (i.e., no "offsite" locations) from July 1, 2013 through February 28, 2018 constituted the initial observations. Information extracted from the database consisted of date, day of week, anesthesiology resident name, attending anesthesiologist name, attending surgeon name, patient age, patient sex, American Society of Anesthesiologists physical status classification (ASA PS), in-patient versus day surgery status, surgery initiation and completion time, and time the patient entered and exited the operating room. Only cases of ASA physical status 1, 2, or 3 patients involving an anesthesia resident and attending anesthesiologist were retained. Transplant, intrathoracic, obstetric, neurosurgical, and otolaryngological procedures were excluded.

During data cleaning, errors were discovered in procedure duration times, emergence times, and resident, surgeon and anesthesiologist names. Duration and emergence times below ten and three 
minutes respectively were considered suspicious and were verified or corrected from original records, as were errors in names that appeared to be alternate spellings of previously included individuals. After these corrections were made, an audit of 30 previously unexamined cases was performed. The audit uncovered errors in one resident name and two ASA classifications, but no additional errors in duration or emergence times.

\section{$\underline{\text { Analysis }}$}

The distributions of continuous variables were examined with descriptive statistics and histograms. Since emergence time had outliers, values greater than 30 minutes were converted to 30 for analysis. The continuous predictors were sufficiently normal to be used in linear regression, but Spearman correlations were used in bivariate analysis to compensate for remaining deviations from normality. T-tests and ANOVAs were performed to measure levels of association between categorical predictors and the outcome variable. These analyses gave a preliminary sense of the data, but do not account for clustering of cases within residents, surgeons, and anesthesiologists, which was addressed in the multivariate models.

A multivariate mixed model was built using a forward selection process in which covariates were added to the model in order of bivariate significance. Variables with $p>0.20$ were removed from the model. After all variables were added, variables with $p>0.05$ were removed from the model. Correlation due to repeated measures on residents was controlled for by modeling resident as a random effect. The effects of surgeons and attending anesthesiologists were entered as fixed factors. The large number of possible combinations rendered an analysis in which all three provider groups were considered as random factors statistically challenging, so only residents 
(the most critical of the three) were analyzed in that way. Surgeons and attending anesthesiologists with fewer than five cases each were grouped. Due to concerns with data reliability, a sensitivity analysis was run removing ASA classification from the final model. All analysis was performed in SAS version 9.4 (Cary, NC).

\section{Results}

A total of 3,072 cases were used in the final analysis. The 63 residents involved with the study participated in 1 to 111 cases each, with training times ranging from 0 to 2.99 years at the time of the case. Procedure duration ranged from $0.02-4.23$ hours. Emergence time before being capped at 30 ranged from 2-193 minutes with a median (IQR) of 12 (8-16). Study cases involved 115 unique surgeons with $1-202$ cases each, and 42 attending anesthesiologists with $1-250$ cases each. After those with fewer than 5 cases each were grouped, there were 73 unique surgeon IDs and 33 anesthesiologist IDs in the final model. See Table 1 for additional case characteristics.

Of the continuous covariates, only procedure duration time was significantly correlated with emergence time $(r=0.145, p<0.001)$. Independent sample $t$-tests indicated that in-patient emergence times were 97 seconds $(95 \% \mathrm{CL}=(69,126))$ longer than out-patient emergence times, and emergence times for male patients were 40 seconds $(95 \% \mathrm{CL}=(14,65))$ longer than those for female patients. One-way ANOVAs indicated significant differences in emergence times by day of the week $(p<0.001)$ and ASA classification $(p=0.012)$. 
Resident training time was not significantly predictive of emergence time in the unadjusted model. However, after controlling for procedure duration time, in-patient status, ASA class, surgeon, and attending anesthesiologist, resident training time became highly significant $(\mathrm{p}<$ 0.001). Day of the week was no longer predictive of emergence times after controlling for surgeon and attending anesthesiologist. In the fully adjusted model, one year of resident training was associated with a reduction in emergence time by 28 seconds $(95 \% \mathrm{CL}=(12,44))$. A onehour increase in procedure time was associated with an increase in emergence time of 34 seconds $(95 \% \mathrm{CL}=(17,50))$. In-patient emergence times were an average of 69 seconds $(95 \% \mathrm{CL}=(36$, 102)) longer than out-patient times. Emergence times for male patients were 37 seconds (95\% $\mathrm{CL}=(10,63))$ longer on average than those for female patients. Patients in ASA class II had average emergence times 41 seconds $(95 \% \mathrm{CL}=(13,69))$ shorter than those in ASA class III, but there was no significant difference between emergence times between ASA classes I and III. Resident covariance was significant as a random intercept $\left(\sigma^{2}=0.748, p=0.001\right)$, but not as a random slope. This suggests that while residents differed in mean emergence times (as reflected in their individual intercepts), the slope of the line relating experience to emergence time did not differ significantly between residents. Removing ASA from the sensitivity model resulted in a less than one second change to the estimated effect of resident training time, indicating that the effect persists regardless of errors in ASA classification.

\section{Discussion}

After statistical modeling was performed, resident level of training was statistically inversely correlated to emergence time. As an example: for an ASA class 3 outpatient female the average emergence time was $11 \mathrm{~min} 54 \mathrm{sec}$ for a starting CA-1 resident. In this model, each year of 
resident training was associated with a reduction in emergence time by 28 seconds. Although a statistically significant correlation was demonstrated, the clinical significance is likely low given the relatively small amount of actual time saved. It should be noted, that other outstanding confounders were likely not fully corrected for, such as surgeon preference for emergence or anesthesia attending supervision at emergence. Additionally, over the course of an anesthesia residency, a resident only reduces their emergence time by approximately 90 seconds on average. The absolute value of this amount of time is unlikely to have impacts on OR efficiency as it pertains to cost saving, as demonstrated in prior studies. The presence or absence of this negative correlation may still offer a metric to help assess resident progression towards clinical competency. Additional limitations of this study include single center data collection and the retrospective nature of the data. The inclusion criteria were designed to exclude cases that may have inaccurate or misrepresentative emergence times (i.e. complex cases, emergent cases, et cetera). Our data also demonstrated a central tendency towards residents earlier in their training median and (IQR) for resident training year $0.69(0.30-1.71)$. This could be due to more senior residents being at off-site rotations or doing more complex cases that were excluded from the sample. Future studies may benefit from targeting specific ranges for resident training year and capturing a given number of cases to better stratify the data by training level.

\section{Limitations}

Data acquisition occurred at a single site. Multi-center review of more anesthesia providers caring for patients in higher volume and variety may provide different findings. ASA PS 4 and 5 patients were excluded to exclude bias, creating a more homogenous group of patients and procedures. This prevented observing advanced residents in higher acuity situations. The same 
applies to excluding transplant, cardiac, intrathoracic, obstetric, neurosurgical, and otolaryngological procedures. Special circumstances could introduce delays beyond control of anesthesiology residents, but this exclusion may mask improving resident efficiency with days of training for these cases. Retrospective analysis of times entered into electronic records leaves room for erroneous or inaccurate data entry and subsequently misled interpretation.

\section{Conclusions}

Emergence time trends collected for individual residents may provide a new body of objective data for assessment of clinical efficiency and growth. This emergence time trend would need to be assessed in combination with other metrics in order to truly establish if a resident is in fact appropriately progressing towards competency. Foreseeably, this data could be made available to program directors and integrated into the processes already in place to assess resident milestone progression and competency. The data could also be correlated with other metrics such as board pass rate and in training exam (ITE) scores in an attempt to validate emergence time as a valid clinical competency correlate.

\section{List of Abbreviations}

ACGME - Accreditation Council for Graduate Medical Education

ASA - American Society of Anesthesiologists

CA - Clinical Anesthesia

$\mathrm{CL}-$ Confidence Limit

IQR - Interquartile Range

IRB - Institutional Review Board

ITE - In training exam

PS - Physical Status

\section{Declarations}


Ethics approval and consent to participate - The Drexel University Institution Review Board (IRB) approved this retrospective review, Protocol \#1701005145. There was no patient consent for a retrospective review not including personal identifying information.

Consent for publication - Not applicable. No individual person's data was collected.

Availability of data and materials - The datasets used and/or analyzed during the current study are available from the corresponding author on reasonable request.

Competing interests - No financial or non-financial competing interests to disclose.

Funding - No funding to disclose.

Authors' contributions - LM is a contributing author. JG is a contributing author and implemented the statistical plan/analysis. CRH is the corresponding author, aiding in data acquisition and primary manuscript writing. MSG is the principal investigator and edited the final manuscript. Acknowledgements - Not applicable.

\section{References}

1. ACGME. “Common Program Requirements.” ACGME Common Program Requirements, ACGME, 2019, www.acgme.org/What-We-Do/Accreditation/Common-Program-Requirements.

2. House, L. M., Calloway, N. H., Sandberg, W. S., \& Ehrenfeld, J. M. (2016). Prolonged patient emergence time among clinical anesthesia resident trainees. Journal of Anaesthesiology, Clinical Pharmacology, 32(4), 446-452.

3. Eappen, S., Flanagan, H., \& Bhattacharyya, N. (2004). Introduction of anesthesia resident trainees to the operating room does not lead to changes in anesthesia-controlled times for efficiency measures. Anesthesiology, 101(5), 1210-1214. 
4. Urman, R. D., Sarin, P., Mitani, A., Philip, B., \& Eappen, S. (2012). Presence of anesthesia resident trainees in day surgery unit has mixed effects on operating room efficiency measures. The Ochsner Journal, 12(1), 25-29. 